\title{
El yodo durante la gestación, lactancia y primera infancia. Cantidades mínimas y máximas: de microgramos a gramos
}

\author{
G. Morreale de Escobar y F. Escobar del Rey \\ Instituto de Investigaciones Biomédicas Alberto Sols, CSIC y UAM. Madrid. \\ (An Esp Pediatr 2000; 53: 1-5)
}

Las hormonas tiroideas, tiroxina (T4) y 3,5,3'-triyodotironina (T3) son necesarias durante todas las fases de la vida para una función normal del sistema nervioso central (SNC). Son especialmente cruciales durante el desarrollo del SNC, pues una insuficiencia de estas hormonas se acompaña de lesiones y defectos neurológicos permanentes e irreversibles.

Ambas hormonas contienen yodo, cuatro átomos por molécula en el caso de la T4, tres en el caso de la T3. Sin yodo no es posible su síntesis, a pesar de lo cual a lo largo de la evolución no han aparecido otras hormonas capaces de sustituirlas y que no tengan esta total dependencia de un elemento, que suele encontrarse en cantidades muy pequeñas fuera del ambiente acuático marino.

En cambio, ha evolucionado una estructura, el folículo tiroideo, capaz de minimizar las consecuencias de un aporte asaz variable del yodo, obtenido en su mayor parte a través de los alimentos y el agua. Es la única estructura endocrina capaz de almacenar estas hormonas en forma de prohormona (la tiroglobulina), con tal eficacia que un adulto, que ha tenido una nutrición adecuada de yodo, puede hacer frente a las necesidades hormonales de su organismo durante varios meses después de iniciarse un período de carencia total del mismo en su alimentación. A su vez, la glándula tiroides del adulto es capaz de evitar las posibles consecuencias nocivas de la producción de un exceso de hormonas tiroideas, que podrían producirse al llegarle cantidades muy altas de yodo. Si embargo, surgen problemas importantes cuando la deficiencia de yodo en la alimentación se hace crónica, o cuando la exposición a un exceso de yodo es muy prolongada, sobre todo cuando esto ocurre durante un período del desarrollo en que la glándula aún no está plenamente preparada para ello. De no resolverse estos problemas, o de resolverse a destiempo, pueden producirse déficit más o menos graves e irreversibles del SNC.
En este breve comentario se intentará definir, con mayor precisión, cuáles son las cantidades mínimas para un desarrollo normal del SNC, y cuáles las que pueden dar lugar a problemas durante el embarazo y primera infancia, períodos en los que tienen lugar en el ser humano fases cruciales de maduración cerebral. Hay, sobre todo con respecto al exceso de yodo, bastantes problemas de índole práctico, tal y como describen con acierto y amplio apoyo bibliográfico Arena y Emparanza en este mismo número ${ }^{1}$.

\section{CANTIDADES MÍNIMAS DE YODO}

Desde que, hace ya una década, fue ratificada por la práctica totalidad de los países del mundo la Declaración Mundial para la Supervivencia, Protección y Desarrollo de la Infancia, así como un Plan de Acción concreto, elaborado por la Convención sobre los Derechos de la Infancia, emanada a su vez de la Cumbre de la Infancia organizada por Naciones Unidas en 1989, se puede afirmar como derecho humano básico de la infancia $^{2}$ que:

1. "Todo niño tiene el derecho a una cantidad adecuada de yodo en su dieta".

2. "Toda madre debe tener una nutrición adecuada de yodo para evitar que el niño tenga un desarrollo mental afectado por una carencia de este micronutriente esencial".

El segundo punto se deriva de la creciente evidencia de que una deficiencia de yodo durante el embarazo puede llevar a concentraciones circulantes de T4 materna insuficientes para un desarrollo armónico del cerebro del feto y el neonato. Las bases científicas y epidemiológicas (que resumimos en un anterior número de esta Revista $^{3}$ ) han llevado a la Organización Mundial de la Salud a declarar que la carencia de yodo es la causa mundial más frecuente de retraso mental y parálisis ce-

Correspondencia: Dra. G. Morreale de Escobar. Instituto de Investigaciones Biomédicas Alberto Sols, CSIC

y UAM. Arturo Duperier, 4. 28029 Madrid.

Correo electrónico: gmorreale@iib.uam.es 
TABLA 1. Ingestas mínimas de yodo, recomendadas a partir de $1992^{6}$

\begin{tabular}{|lcc|}
\hline Grupo & Edad & $\mu$ g de I /día \\
\hline Prematuros & & $>30 \mu \mathrm{g} / \mathrm{kg} / \mathrm{dí} a$ \\
Niños & $0-5$ meses & 90 \\
& $6-12$ meses & 90 \\
& $1-3$ años & 90 \\
& $4-6$ años & 90 \\
Adultos & $7-10$ años & 120 \\
Mujeres embarazadas & & 150 \\
Mujeres lactantes & & $230^{*}$ \\
\end{tabular}

"Estas son las recomendaciones mínimas en Alemania. Recientemente (después de 1992) el ICCIDD (International Council for the Control of Iodine Deficiency Disorders) ha elevado a 200-300 $\mu \mathrm{g}$ /día las ingestas recomendadas para las embarazadas

rebral prevenibles, afectando en mayor o menor grado el desarrollo y bienestar de unos 1.600 millones de los actuales habitantes de nuestro planeta. Algunos de ellos viven en España, donde se ha constatado la persistencia de deficiencia de yodo en las 14 comunidades autónomas en las que se han realizado estudios recientes al respecto.

En la tabla 1 aparecen las recomendaciones actuales sobre las cantidades mínimas de yodo que se consideran necesarias durante diferentes fases de la vida. Las cantidades han ido elevándose a medida que se han ido teniendo datos epidemiológicos más precisos, y un conocimiento más completo de las diferencias en la fisiología tiroidea a distintas edades. Nótese que las necesidades de yodo de niños prematuros, de neonatos y niños pequeños son notablemente más altas de lo que se deduciría, sobre la base de su peso corporal, de las definidas para escolares y adultos. En el caso de niños prematuros, los preparados humanizados comercializados para ellos no contenían yodo suficiente, pero en los últimos años su contenido se ha ido haciendo más acorde con los requerimientos ${ }^{4,5}$.

También han ido aumentando las cantidades recomendadas durante el embarazo, a medida que se han completado los estudios realizados en Europa. En nuestro país, concretamente en la Comunidad Autónoma de Madrid, hemos observado que las embarazadas necesitan un suplemento de $250-300 \mu \mathrm{g} /$ día para que puedan alcanzar concentraciones óptimas de T4 libre circulante, y para no desarrollar bocio durante el embarazo ${ }^{7,8}$.

Dada la gran variabilidad del contenido en yodo de los alimentos de procedencia no marina, se recomienda asegurar estas cantidades mínimas mediante la suplementación de la dieta con sal yodada ${ }^{2}$. En España la legislación contempla la yodación de sal refinada de mesa en $60 \mu \mathrm{g} \mathrm{I} / \mathrm{g}$ sal ( $60 \mathrm{mg} / \mathrm{kg} ; 60$ ppm). El uso habitual de esta sal yodada (que no incluye la sal marina, a no ser que el envase especifique que está yodada), parece suficiente para gran parte de la población. Pero quedan precisamente excluidos los niños prematuros y lactantes y las mujeres embarazadas, que constituyen la parte de la población más vulnerable; los primeros tendrían que ingerir casi $2 \mathrm{~g}$ de sal yodada al día, y sus madres $5 \mathrm{~g} /$ día. Como esto no ocurre, por las recomendaciones actuales de evitar o restringir el uso de sal en dichos grupos de la población, se impone asegurar las cantidades mínimas de yodo mediante la suplementación diaria controlada. No habiendo en la farmacopea española actual preparados de yoduro o yodato potásico (en forma de tabletas, gotas o grageas) adecuados para ello, hay que recurrir a preparados polivitamínicos y minerales que sí lo contienen, como Calcinatal ${ }^{\circledR}$, Multicentrum ${ }^{\circledR}$, Superdyne ${ }^{\circledR}$ y Micebrina ${ }^{\circledR}$. Cuando la madre ha tomado este suplemento durante todo el embarazo y sigue tomándolo durante la lactancia, su leche contiene las cantidades de yodo que necesita su hijo, sea o no prematuro. Pero en el caso de no ser posible la lactancia materna, habrá que recurrir a los preparados que estén adecuadamente enriquecidos con este micronutriente 5 . No hemos encontrado información sobre el contenido en yodo de los alimentos preparados para la alimentación de los niños cuando dejan la lactancia, por lo que la suplementación de su dieta con preparados polivitamínicos y minerales podría resultar aconsejable.

Con frecuencia se expresa el temor de que, cuando la ingesta de yodo de la mujer ya era buena antes del embarazo, una suplementación de su dieta con 250-300 $\mu \mathrm{g} /$ día podría resultar excesiva y dañina. No hay base alguna para tal temor, ya que una ingesta de 1-2 mg diarios de yodo es frecuente en algunas poblaciones que consumen algas marinas, como algunas de Japón ${ }^{9}$, sin efectos nocivos. Medidas de yoduria en embarazadas de Chile, por ejemplo, sugieren ingestas de 500-700 $\mu_{\mathrm{g}}{ }^{10}$, también sin efectos negativos. Como éste es un punto de gran importancia, la Organización Mundial de la Salud encomendó su estudio a un comité internacional de expertos, de cuyas reuniones emanó el documento que demuestra que incluso el uso de aceites yodados (p. ej., Lipiodol ${ }^{\circledR}$ ), utilizado como medida de urgencia para erradicar la deficiencia de yodo en países que no tienen establecida una adecuada red de distribución de sal yodada, está exento de problemas para la embarazada y el desarrollo de su feto ${ }^{11}$. Contrasta esto con los graves problemas relacionados con una ingesta materna deficiente en yodo durante el período de desarrollo fetal (tabla 2$)^{12}$, problemas que se erradican con su utilización ${ }^{13,14}$. Debe tenerse en cuenta que $1 \mathrm{ml}$ del aceite yodado empleado habitualmente (Lipio$\mathrm{dol}^{\circledR}$ ) contiene $380 \mathrm{mg}$ de I (;380.000 $\mu \mathrm{g}$ !) y las dosis empleadas suelen ser de $2 \mathrm{ml}$, o más. Aunque se administre de una sola vez, por vía oral o intramuscular, y se retenga en el músculo y tejido graso, el yodo se va liberando paulatinamente. Pero, obviamente, lo hace en cantidad superior a lo que ingeriría una mujer que recibiese 300 $\mu \mathrm{g} /$ día durante todo el embarazo y la lactancia (110 mg). 


\begin{tabular}{|c|c|}
\hline $\begin{array}{l}\text { Periodo en que } \\
\text { se padece la } \\
\text { deficiencia de yodo }\end{array}$ & Consecuencias principales \\
\hline Feto & $\begin{array}{l}\text { Mayor número de abortos } \\
\text { Nacidos muertos } \\
\text { Anomalías congénitas } \\
\text { Mayor mortalidad perinatal } \\
\text { Mayor mortalidad infantil } \\
\text { Cretinismo neurológico } \\
\text { Deficiencia mental } \\
\text { Sordomudez } \\
\text { Diplejía, tetraplejía espástica } \\
\text { Estrabismo } \\
\text { Cretinismo mixedematoso } \\
\text { Enanismo } \\
\text { Deficiencia mental } \\
\text { Retraso mental de los habitantes } \\
\text { aparentemente normales } \\
\text { Mayor susceptibilidad en caso } \\
\text { de accidentes nucleares* }\end{array}$ \\
\hline Recién nacidos & $\begin{array}{l}\text { Defectos psicomotores } \\
\text { Bocio neonatal } \\
\text { Hipotiroidismo neonatal } \\
\text { Mayor susceptibilidad en caso } \\
\text { de accidentes nucleares* }\end{array}$ \\
\hline $\begin{array}{l}\text { Niños y } \\
\quad \text { adolescentes }\end{array}$ & $\begin{array}{l}\text { Bocio } \\
\text { Hipotiroidismo juvenil } \\
\text { Deterioro de las facultades mentales } \\
\text { Retraso en el desarrollo somático } \\
\text { Mayor susceptibilidad en caso } \\
\text { de accidentes nucleares* }\end{array}$ \\
\hline Adultos & $\begin{array}{l}\text { Bocio y sus complicaciones } \\
\text { Hipotiroidismo } \\
\text { Deterioro de las facultades mentales } \\
\text { Hipotiroidismo por carencia de yodo } \\
\text { Mayor susceptibilidad en caso } \\
\text { de accidentes nucleares* } \\
\text { Propensión a hipertiroidismo al } \\
\text { instaurarse medidas profilácticas }\end{array}$ \\
\hline
\end{tabular}

*Se debe a una mayor avidez de la glándula deficiente en yodo para concentrarlo, incluidos los isótopos radiactivos del mismo, que son liberados en grandes cantidades durante los accidentes nucleares.

Toda la patología tiroidea, incluido el cáncer de tiroides, está aumentada en las zonas de deficiencia de yodo, y a todas las edades.

\section{CANTIDADES EXCESIVAS DE YODO}

Se han descrito casos de grandes bocios en neonatos de madres que usaron sistemáticamente compuestos yodados durante el embarazo (jarabes yodados para la tos, desinfectantes yodados, etc., que contenían gramos de yodo $)^{15}$, o que recibieron contrastes yodados para amniofetografía ${ }^{16}$. En algunos casos se produjo la muerte por asfixia. En la mayoría de los otros se produjo un bloqueo de la función tiroidea del feto y el neonato, cuyos efectos pueden prolongarse durante meses, precisamente durante períodos importantes de maduración cerebral. Debe tenerse en cuenta que para el desarrollo del cerebro durante los primeros años de vida no hay diferencia entre un estado de hipotiroidismo permanente y uno transitorio, pues en ambos casos el niño requiere tratamiento con T4. En el caso de un bloqueo por yodo, el tratamiento debe prolongarse por lo menos hasta que se haya normalizado la yoduria. Por eso parece muy oportuno el artículo de Arena y Emparanza en este número de la Revista ${ }^{1}$, pues parece que en muchas consultas ginecológicas y en maternidades españolas no se ha eliminado totalmente el uso de antisépticos yodados, como el Betadine ${ }^{\circledR}$, que contiene povidona yodada al 10\%. Cuando hace dos décadas se extendieron por toda España los programas para la Detección Precoz del Hipotirodismo Congénito, se advirtió a todas las maternidades sobre los graves inconvenientes del uso de estos antisépticos, pero al parecer con el tiempo esto se ha ido olvidando, encontrándonos en varios congresos recientes que muchos de los asistentes no conocían su prohibición.

También puede ocurrir que la prohibición sí se conozca, pero se haya interpretado erróneamente por una información inexacta. En algunos casos, se evita efectivamente el empleo de povidona yodada para desinfectar al recién nacido, sobre todo si es prematuro, pero sólo hasta después de tomar la muestra de sangre del talón que se envía al Centro de Detección Precoz de Hipotiroidismo Congénito, en la creencia errónea de que su uso "falsea las pruebas" por causas analíticas. A partir de ese momento, se utiliza libremente. En realidad, no es que el uso de la povidona yodada "falsee las pruebas"; lo que hace es provocar un estado de hipotiroidismo que no existía antes de su aplicación. "Falsea" en cuanto no se trata de un hipotiroidismo congénito permanente, sino de uno transitorio. Pero, como se ha indicado más arriba, estos niños requieren tratamiento posnatal con T4 mientras dure el hipotiroidismo, al igual que los niños con hipotiroidismo congénito permanente, si queremos evitar los déficit mentales permanentes que acompañan al hipotiroidismo perinatal tratado tardíamente.

Este efecto nocivo de los desinfectantes yodados administrados a la madre y/o al recién nacido se debe a que en la glándula tiroides del feto y en la del neonato aún no han madurado plenamente los mecanismos de autorregulación tiroidea ${ }^{17}$, que en el adulto permiten obviar los riesgos de una producción excesiva de hormonas tiroideas al producir un aumento la cantidad de yodo disponible.

En el individuo adulto sin patología tiroidea subyacente, el exceso de yodo no produce un bloqueo prolongado de la función tiroidea, ya que se han desarrollado "mecanismos de escape" que se vuelven operativos antes de que disminuyan las concentraciones de T4 y T3 por debajo de las normales. No se conoce bien cómo se ponen en marcha dichos "mecanismos 


\begin{tabular}{|c|c|c|}
\hline \multicolumn{3}{|c|}{$\begin{array}{l}\text { TABLA 3. Concentración de yodo en diferentes } \\
\text { medicamentos, desinfectantes y contrastes } \\
\text { radiológicos de uso muy extendido, } \\
\text { y de } 1 \mathrm{~g} \text { de sal yodada }\end{array}$} \\
\hline & $\begin{array}{l}\text { Contenido } \\
\text { en yodo }\end{array}$ & $\begin{array}{l}\text { Contenido } \\
\text { en yodo } \\
\text { frente a } \\
150 \mu \mathrm{g} / \mathrm{dia}\end{array}$ \\
\hline Sal yodada & $60 \mu \mathrm{g} / 1 \mathrm{~g}$ & $0,4 \mathrm{x}$ \\
\hline Amiodarona & $\begin{array}{l}7.500 \mu \mathrm{g} / \\
\text { comprimido }\end{array}$ & $50 x$ \\
\hline \multicolumn{3}{|l|}{ Desinfectantes } \\
\hline $\begin{array}{l}\text { Solución de Lugol } \\
\text { Betadine (povidona }\end{array}$ & $126.000 \mu \mathrm{g} / \mathrm{ml}$ & $840 \mathrm{x}$ \\
\hline yodada) & $10.000 \mu \mathrm{g} / \mathrm{ml}$ & $67 x$ \\
\hline Yoduro sódico al $10 \%$ & $85.000 \mu \mathrm{g} / \mathrm{ml}$ & $570 x$ \\
\hline Vioformo/clioquinol & $12.000 \mu \mathrm{g} / \mathrm{ml}$ & $80 \mathrm{x}$ \\
\hline Enterovioformo & $\begin{array}{l}120.000 \mu \mathrm{g} / \\
\text { comprimido }\end{array}$ & $800 \mathrm{x}$ \\
\hline \multicolumn{3}{|l|}{ Contrastes radiológicos } \\
\hline Hexabrix & $320.000 \mu \mathrm{g} / \mathrm{ml}$ & $2.100 \mathrm{x}$ \\
\hline Oragrafin & $\begin{array}{l}308.000 \mu \mathrm{g} / \\
\text { cápsula }\end{array}$ & $2.050 \mathrm{x}$ \\
\hline Lipiodol & $380.000 \mu \mathrm{g} / \mathrm{ml}$ & $2.500 \mathrm{x}$ \\
\hline Renografin & $370.000 \mu \mathrm{g} / \mathrm{ml}$ & $2.500 \mathrm{x}$ \\
\hline Telepaque & $333.000 \mu \mathrm{g} / \mathrm{ml}$ & $2.200 \mathrm{x}$ \\
\hline
\end{tabular}

"150 mg/día suele ser la ingesta diaria mínima considerada adecuada para jóvenes y adultos, excluyendo mujeres embarazadas y lactantes.

de escape", pero sí se sabe que aún no son plenamente operativos en el recién nacido, y lo son tanto menos cuanto menor sea su edad gestacional. Éste es el motivo de la gran frecuencia con que se bloquea la glándula tiroidea del niño prematuro, al enfrentarse a cantidades de yodo que son toleradas perfectamente por un adulto.

El riesgo de un bloqueo de la glándula del neonato no sólo aumenta en el caso de que haya nacido prematuramente, sino que depende también en gran medida de la ingesta de yodo materna. Cuando ésta ha sido insuficiente, el aclaramiento de yoduro por la glándula tiroides del niño, así como su tamaño, aumentan rápidamente y de forma considerable. Pero al volver a llegar yodo en cantidades adecuadas, o altas, no disminuye rápidamente la excesiva vascularización de la glándula, ni la captación aumentada de yodo. Hay un desfase temporal importante, por lo que al llegarle una cantidad excesiva de yodo, la glándula acumula una proporción mayor que en el caso de un recién nacido sin carencia anterior de yodo. Esto contribuye a que se observe un bloqueo de la glándula con dosis de yodo que no resultan excesivas cuando la población está bien nutrida. Los efectos bloqueadores del yodo se ven potenciados cuando se superponen una cierta deficiencia de yodo y la inmadurez de la glándula del prematuro. Por eso, en muchos países europeos (España incluida) el hipotiroidismo neonatal por exceso de yodo es mucho más frecuente que en Japón y en los Estados Unidos, donde la ingesta de yodo de la población es más alta.
Esta llamada de atención sobre los peligros de un exceso iatrogénico de yodo durante el embarazo y el período posnatal no debe, en manera alguna, utilizarse como justificación para dejar de instaurar medidas profilácticas que eviten la carencia de yodo durante el desarrollo fetal y posnatal.

Siempre debe tenerse en cuenta la gran diferencia existente entre las cantidades mínimas necesarias y las cantidades de yodo potencialmente nocivas. Considérese que las cantidades mínimas necesarias se expresan en cientos de $\mu \mathrm{g}$ de yodo (200-300 $\mu \mathrm{g}$ en la embarazada). La cantidad de povidona yodada en $1 \mathrm{ml}$ de Betadine ${ }^{\circledR}$ es de $100 \mathrm{mg}$ (!!), lo que equivale a unos $100.000 \mu \mathrm{g}$. En cualquier aplicación de povidona yodada se emplea un volumen de desinfectante muy superior a $1 \mathrm{ml}$. Lo mismo ocurre cuando se usan contrastes yodados (tabla 3).

Cualquier neonato, sobre todo si es prematuro, que inevitablemente tiene que someterse a pruebas diagnósticas o quirúrgicas que hagan imprescindible la administración de contrastes yodados, puede padecer un bloqueo de la función tiroidea como consecuencia de la intervención, y entrar en un estado de hipotiroidismo. Por eso, y por el frecuente retraso en el pleno funcionamiento de los mecanismos de retroalimentación negativa hipófisis-tiroides característicos de esa edad, se recomienda que no sólo se envíen al Centro de Detección Precoz de Hipotiroidismo Congénito las muestras de sangre tomadas a los pocos días del nacimiento, sino cada vez que se hayan administrado contrastes yodados. No debe darse el alta al paciente sin tener pruebas bioquímicas de que no padece hipotiroidismo, aunque sea adquirido y transitorio.

Las cantidades de yodo de la mayoría de los contrastes yodados son incluso superiores a las mencionadas para la povidona yodada (tabla 3). A veces no se tiene conciencia de que el recién nacido está recibiendo esta sobredosis: la mera inserción de catéteres (no radioopacos) para alimentación parenteral conlleva la inyección de contraste en cantidades muy pequeñas, pero suficientes para bloquear la función tiroidea ${ }^{18}$, por lo que deben sustituirse por catéteres radioopacos.

En resumen, en el caso de la mujer embarazada, el lactante y el neonato (sea éste prematuro o a término), hay que tener presente que:

1. Tienen derecho a que se le asegure el yodo necesario para el desarrollo óptimo de su cerebro.

2. Es sumamente improbable que reciba un exceso nocivo de yodo a través de la alimentación y el uso de suplementos polivitamínicos y minerales que lo contengan.

3. Puede ser el personal sanitario el primer responsable de que se vean expuestos a dosis excesivas, que provienen siempre del uso de diferentes medicamentos, desinfectantes yodados y contrastes radiológicos. 


\section{Bibliografía}

1. Arena Ansotegui J, Emparanza Knörr JI. Los antisépticos yodados no son inocuos. An Esp Pediatr 2000; 53: 25-29.

2. Escobar del Rey F, Morreale de Escobar G. Yodación universal de la sal: un derecho humano de la infancia. Endocrinología 1998; 45: 4-16.

3. Morreale de Escobar G. Interrelaciones materno-fetales de las hormonas tiroideas. An Esp Pediatr 1999; 50 (Supl 125): 3643

4. Ares S, Quero J, Duran S, Presas MJ, Herruzo R, Morreale de Escobar G. Iodine content of infant formulas and iodine intake of premature babies: high risk of iodine deficiency. Arch Dis Child (Fetal Neonatal) 1994; 71: F184-F191.

5. Ares S, Morrreale de Escobar G, Quero J. Lactancia artificial $\mathrm{y}$ deficiencia de yodo en el niño prematuro. An Esp Pediatr 1999; 50 (Supl 125): 47-51.

6. Delange F, Dunn JT, Glinoer D. Specific recommendation on iodine nutrition for mothers and infants in Europe. En: Delange F, Dunn JT, Glinoer D, editores. Iodine Deficiency in Europe. Nueva York: Plenum Press, 1993; 478-479.

7. De Santiago J, Pastor I, Escobar del Rey F, Morreale de Escobar G. Thyroid function in pregnant women from an area with mild (grade I) iodine deficiency [resumen 126]. J Endocrinol Inv 1999; 22 (Supl 6): 68.

8. De Santiago García J, Pastor I, Escobar del Rey F, Morreale de Escobar G. Deficiencia de yodo y función tiroidea de la embarazada. 41 Congreso Nacional de la Sociedad Española de Endocrinología y Nutrición. Málaga, 1999.

9. Katamine S, Mamiya K, Sekimoto N, Hoshino N, Totsuka K, Naruse A et al. Iodine contant of various meals currently consumed by urban Japanese. J Nutr Sci Vitaminol 1986; 32: 487492 .
10. Liberman CS, Pino SC, Fang SL, Braverman LE, Emerson $\mathrm{CH}$. Circulating iodide concentrations during and after pregnancy. J Clin Endocrinol Metab 1998; 83: 3545-3549.

11. WHO. Iodized oil during pregnancy. Safe use of iodized oil to prevent iodine deficiency in pregnant women: a WHO statement. Bull WHO 1996; 74: 1-3.

12. Hetzel BS. Historical development of concepts of brain-thyroid relationships. En: Stanbury JB, editor. The damaged brain of iodine deficiency. Elmsford, NY: Cognizant Communication Co., 1994; 1-8.

13. Pharoah POD, Buttfield IH, Hetzel BS. Neurological damage to the fetus resulting from severe iodine deficiency during pregnancy. Lancet 1971; 13: 308-311.

14. Pretell EA, Cáceres A. Impairment of mental development by iodine deficiency and its correction. A retrospective view from studies in Peru. En: Stanbury JB, editor. The damaged brain of iodine deficiency. Elmsford, NY: Cognizant Communication Co., 1994; 187-192.

15. Carswell F, Kerr MM, Hutchison JH. Congenital goitre and hypothyroidism produced by maternal ingestion of iodides. Lancet 1970; 13: 1242-1247.

16. Rodesh F, Camus M, Ermans AM, Dodion J, Delange F. Adverse effects of amniofetography on fetal thyroid function. Amer J Obstet Gynecol 1976; 126: 723-726.

17. Delange F, Bourdoux P, Ermans AM. Transient disorders of thyroid function and regulation in preterm infants. En: Delange F, Fisher DA, Malvoux P, editores. Pediatric thyroidology. Basilea: S Karger AG, 1985; 14: 369-393.

18. Ares S, Pastor I, Quero J, Morreale de Escobar G. Thyroid complications, including overt hypothyroidism, related to the use of non-radiopaque silastic catheters for parenteral feeding in prematures requiring injection of small amounts of an iodinated contrast medium. Acta paediatr 1995; 84: 579-581. 